\title{
S. R. Suleiman, The Némirovsky Question. The Life, Death and Legacy of a Jewish Writer in 20th-Century France
}

\section{Elena Quaglia}

\section{(2) OpenEdition Journals}

\section{Edizione digitale}

URL: http://journals.openedition.org/studifrancesi/10045

DOI: $10.4000 /$ studifrancesi. 10045

ISSN: 2421-5856

\section{Editore}

Rosenberg \& Sellier

\section{Edizione cartacea}

Data di pubblicazione: 1 août 2017

Paginazione: 395-396

ISSN: 0039-2944

\section{Notizia bibliografica digitale}

Elena Quaglia, «S. R. Suleiman, The Némirovsky Question. The Life, Death and Legacy of a Jewish Writer in 20th-Century France», Studi Francesi [Online], 182 (LXI | II) | 2017, online dal 01 août 2017, consultato il 07 janvier 2021. URL: http://journals.openedition.org/studifrancesi/10045 ; DOI: https://doi.org/ ERREUR PDO dans /localdata/www-bin/Core/Core/Db/Db.class.php L.34 : SQLSTATE[HY000] [2006] MySQL server has gone away

Questo documento è stato generato automaticamente il 7 janvier 2021.

\section{(†)

Studi Francesi è distribuita con Licenza Creative Commons Attribuzione - Non commerciale - Non opere derivate 4.0 Internazionale. 


\title{
S. R. Suleiman, The Némirovsky Question. The Life, Death and Legacy of a Jewish Writer in 20th-Century France
}

\author{
Elena Quaglia
}

\section{NOTIZIA}

SUSAN RUBIN SULEIMAN, The Némirovsky Question. The Life, Death and Legacy of a Jewish Writer in $20^{\text {th }}$-Century France, New Haven and London, Yale University Press, 2016, 377 pp.

1 Il titolo dell'ultimo libro di Susan Rubin Suleiman, The Némirovsky Question, allude a un'altra annosa "questione", oggetto di numerosissimi saggi, da Marx a Sartre: quella questione ebraica che i nazisti vollero risolvere con lo sterminio. Suleiman riflette nella sua monografia sull'aspetto più controverso e per questo più affascinante della vita $\mathrm{e}$ dell'opera di Irène Némirovsky, cioè sul suo rapporto con l'identità ebraica. La saggista tenta quindi di dare risposta a una domanda spesso posta dalla critica, soprattutto dal 2004, con la pubblicazione postuma di Suite française: Némirovsky è un'ebrea che odia se stessa?

2 Nel primo capitolo del saggio, Suleiman analizza il concetto di "odio di sé", ricostruendone la fortuna. I concetti di ambiguità e ambivalenza (cfr. p. 21) sembrano infine essere più atti a indagare l'atteggiamento nei confronti dell'identità ebraica, non soltanto di Némirovsky ma anche di molti suoi contemporanei nell'epoca tra le due guerre. In particolare, una questione centrale è quella del rapporto tra gli "Israeliti", vale a dire gli ebrei ricchi, ben inseriti nella società francese, e gli ebrei poveri, recentemente immigrati dall'Est Europa. Grazie all'analisi della novella Fraternité, Suleiman dimostra brillantemente come l'incontro tra un Ebreo e un Israelita faccia nascere in quest'ultimo il terrore di assomigliare a quell'altro da sé di cui pure condivide il cognome. Secondo la saggista americana, in questa novella emergerebbe 
una visione dell'identità ebraica caratterizzata dall'ansia e dalla paura dell'esclusione, ricorrente nell'opera di Némirovsky.

3 Nel secondo capitolo, Suleiman conduce un'analisi molto documentata del percorso della romanziera nel campo letterario, scegliendo di evitare una lettura sociologica talvolta troppo deterministica e già alla base della monografia dedicata a Némirovsky da Angela Kershaw. La saggista adotta così un approccio simile a quello della Microstoria: evitando ogni visione prospettica ex-post («backshadowing», p. 47), essa analizza di volta in volta le scelte della scrittrice, confrontandole a quelle dei suoi contemporanei. In particolare, la romanziera di origini russe si lega a un ambiente letterario istituzionale, inserendosi, unica donna con Colette, in un contesto fortemente maschile: questa posizione nel campo letterario la porta a confrontarsi con un antisemitismo sempre più diffuso nelle riviste degli anni Trenta. Suleiman si interessa in particolare alla collaborazione di Némirovsky a «Gringoire», mai interrotta, anche dopo la campagna di Béraud contro Blum nel 1936, a differenza di quanto fecero Kessel o Gary. Se i biografi dell'autrice, così come le sue figlie, rilevavano la distanza tra le pagine politiche e le pagine letterarie della rivista, l'analisi di Suleiman smentisce tale prospettiva, insistendo sulle necessità economiche di Némirovsky. La saggista americana porta inoltre alla luce nuovi documenti riguardanti le richieste di cittadinanza francese da parte della romanziera e la sua conversione al cattolicesimo.

seguito, nel corso del terzo capitolo, Suleiman si concentra sugli ultimi anni della carriera di Némirovsky, tra l'inizio della Seconda Guerra Mondiale e la sua deportazione nel 1942. Anche in questa sezione la saggista tenta di mostrare tutti i complessi moventi delle scelte della romanziera, apportando nuova documentazione biografica.

5 Nella seconda parte del saggio, Suleiman si dedica all'analisi letteraria dei testi di Irène Némirovsky che presentano personaggi di origine ebraica. Sulla scia del suo lavoro sul roman à thèse, nel quarto capitolo, la saggista si interroga sulla presenza dell'antisemitismo in un racconto di finzione, affermando infine che nell'opera di Némirovsky il manicheismo stereotipico non è così nettamente delineato come nei testi antisemiti. In particolare, l'universo ebraico, secondo Suleiman, è raramente confrontato all'universo francese, ma è visto dall'interno. La romanziera si concentra soprattutto su coppie di personaggi ebrei: l'uno è lo specchio rovesciato dell'altro, uno specchio che riporta alle origini e alle angosce che vi si collegano.

6 Il quinto capitolo focalizza l'analisi del testo su due figure fondamentali dell'opera némirovskiana: Hélène, la protagonista autobiografica del romanzo Le vin de solitude $\mathrm{e}$ Ada, la pittrice di Les chiens et les loups. Attraverso queste due artiste ebree, la romanziera mette in scena la sua postura ambivalente, la sua «in-betweenness» (p. 184), tra volontà di assimilazione e desiderio di distinzione.

7 Nella terza parte del saggio, Suleiman si concentra sul periodo che segue la deportazione di Némirovsky e in particolare sulla vita delle sue figlie, sopravvissute alla Shoah e rimaste orfane. Dopo molte pagine nelle quali ricostruisce gli anni di guerra, la fuga delle due bambine e la loro situazione nell'immediato dopoguerra, che occupano la quasi totalità del sesto capitolo, Suleiman riprende le riflessioni già sviluppate nel suo libro intitolato Crises of memory, riguardanti i bambini sopravvissuti alla Shoah, la cosiddetta generazione 1,5 .

8 Nel settimo capitolo, Suleiman intreccia brillantemente l'analisi della fortuna postuma dell'autrice con la storia delle sue figlie e dei suoi nipoti e pronipoti. Molte pagine sono 
in particolare dedicate a Le Mirador, l'autobiografia «rêvée» di Némirovsky scritta da Elisabeth Gille, figlia minore dell'autrice, nel tentativo di comprendere le scelte della madre. Suleiman ripercorre inoltre la storia di Suite française, decostruendo alcuni miti: la saggista formula infatti l'ipotesi che la celebre valigia contenente il manoscritto inedito sia entrata in possesso delle figlie di Némirovsky soltanto nel 1956 e non subito dopo la guerra. La conclusione del saggio apre un breve scorcio sull'epoca attuale: Suleiman si interroga infatti sulla questione di un rinnovato antisemitismo contemporaneo.

9 La monografia sarà pubblicata in francese nel 2017 da Albin Michel, editore di Némirovsky stessa. Notevolmente documentata, tale ricerca apre nuove prospettive per lo studio della vita e dell'opera dell'autrice. Le analisi dei testi mostrano le contraddizioni di una visione dell'identità ebraica non riducibile alla mera etichetta di "odio di sé", ma ascrivibile alla medesima complessità manifestata dalle opere di Kafka o di Proust. Susan Rubin Suleiman ha il merito di riprendere le riflessioni svolte in una lunga carriera di critica letteraria e di applicarle in maniera convincente allo studio dei testi di Irène Némirovsky e della figlia, Elisabeth Gille. Le future ricerche non potranno che beneficiare di questa ricostruzione appassionata e appassionante di una vita e di un'opera e dei loro riflessi post-genocidari. 